\title{
The One-dimensional Property of Sr/Si(111)-3×2 Reconstructed Surface
}

\author{
Wenhan DU ${ }^{a}$, Jingjing YANG, Yu ZHAO, Chao XIONG, Jin XIAO, Jinxiang MA and \\ Xifang ZHU \\ Changzhou Institute of Technology, ChangZhou, JiangSu Province, China \\ aduwh@czu.cn, 1
}

\begin{abstract}
The electronic and geometric structures of $\mathrm{Sr} / \mathrm{Si}$ surface were investigated by Scanning Tunneling Microscopy. One-dimensional(1D) atomic concerted motion phenomenon was observed on this surface. Three reasons contribute to this phenomenon: First, low metal coverage of $1 / 6$ monolayer with low energy occupation sites; second, weak interaction between $\mathrm{Sr}$ and Si substrate; third, surface vacancy formed in the high temperature annealing process and thermal fluctuation energy at room temperature. This findings lead to the conclusions that $(3 \times 2)$ reconstruction of $\mathrm{Sr} / \mathrm{Si}$ system behave typical one-dimensional diffusion property at room temperature.
\end{abstract}

\section{Introduction}

$\mathrm{Sr} / \mathrm{Si}$ reconstruction surface play an important role in the growth of high k oxides, such as $\mathrm{SrTiO} 3$ on Si substrate in semiconductor industry to reduce gate leakage current with the CMOS device dimension decreasing.

Different reconstruction structures, such as $(2 \times 3) \mathrm{Sr} / \mathrm{Si}$ and $(2 \times 1) \mathrm{Sr} / \mathrm{Si}$ have been reported for the $\mathrm{Si}(100)$ substrate, and plausible reconstruction structures have been proposed ${ }^{[1-10]}$. As for $\mathrm{Si}(111)$ substrate, when $\mathrm{Sr}$ atoms adsorbed on this surface, (3x2)-Sr/Si(111) reconstruction structure has been observed ${ }^{[7]}$. However we noticed that $1 \times 3$ spots in the LEED pattern was observed, and $3 \times 2$ spots was weak. What arouse this contradiction, there are still lack plausible explanations.

Here, we use STM and STS to investigate $(3 \times 2)-\mathrm{Sr} / \mathrm{Si}(111)$ surface, and propose the plausible reason to compensate the difference between STM and LEED patterns.

\section{Experiment}

The experiments were performed in an ultra-high vacuum system with base pressure of $4.0 \times 10^{-8} \mathrm{~Pa}$, and equipped with a VT STM (MATRIX, Omicron GmbH). High resistive p-type $\operatorname{Si}(111)$ wafers were used as substrates. A pulsed laser deposition (PLD) technique

* Corresponding author: duwh@czu.cn, 
was used to deposit $\mathrm{SrO}$ ultra-thin film with nominal thickness of $1.0 \mathrm{~nm}$ on $\mathrm{Si}(111)-7 \times 7$ substrate at room temperature. After deposition, the sample was annealed at $700^{\circ} \mathrm{C}$ for about $1 \mathrm{~h}$, during this process the $\mathrm{O}$ atoms in the $\mathrm{SrO}$ will react with $\mathrm{Si}$ and forming volatile $\mathrm{SiO}^{8}$, thus leaving $\mathrm{Sr} / \mathrm{Si}(111)$ reconstruction structure. Scanning tunneling microscopy measurements were performed at room temperature (RT).

\section{Results and Discussions}

\subsection{Geometric Structure of Sr/Si(111)-3x2 Surface}

In the large scale STM image which shown in fig.1 (a), it can be noticed that $\mathrm{Sr} / \mathrm{Si}(111)-3 \times 2$ surface reconstructed well ordered. A selected area were shown in the fig. 1 (b), in this high resolution image two type atom rows appear: One was clear rows with atomic resolution, the other was blur rows with different length. The clear rows is characterized by a $3 \times 2$ unit cell (parallelogram) with a $3 \mathrm{a}_{0}$ between the neighboring rows and $2 \mathrm{a}_{0}$-period along the rows, here $\mathrm{a}_{0}=3.84 \AA$ is the lattice constant of $\mathrm{Si}(111)$. For clarified whether the $\mathrm{Sr} / \mathrm{Si}(111)-3 \times 2$ surface has bias polarity dependence or not, we measured high resolution STM image shown in fig.1(c), during the measurement we change the bias polarity twice to identify the difference between filled state and empty state images. We observed one prominent circle protrusion appearing in the empty state image of $1.5 \mathrm{~V}$, and two protrusions appearing in the filled state of $-1.5 \mathrm{~V}$ in one unit cell. The bright protrusion in the empty state was situated in the middle of two protrusions in filled state image.
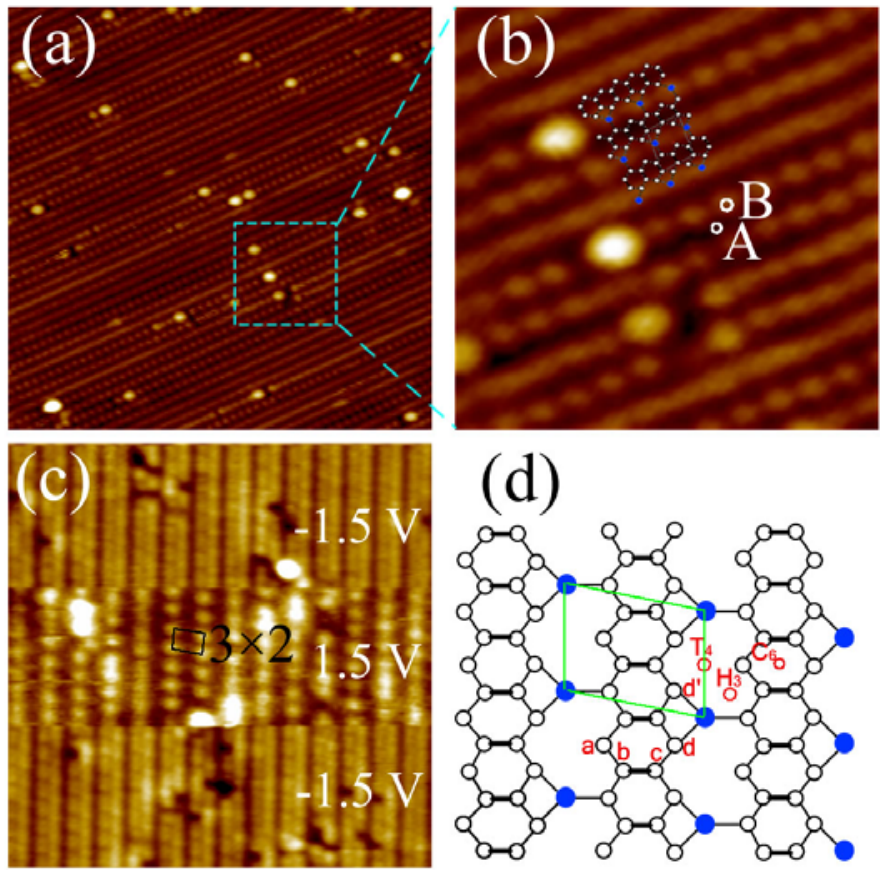

Fig. 1 (a) $\mathrm{Sr} / \mathrm{Si}(111)-3 \times 2$ surface with condition of $\mathrm{V}_{\text {bias }}=3.5 \mathrm{~V}_{\mathrm{I}_{\mathrm{set}}}=20 \mathrm{pA} \quad 40 \times 40 \mathrm{~nm}^{2}$. (b) Enlarge STM image from (a), $10 \times 10 \mathrm{~nm}^{2}$. (c) High resolution dual bias STM image, $\pm 1.5 \mathrm{~V}$ and tunneling current of $10 \mathrm{pA}, 15 \times 15 \mathrm{~nm}^{2}$. (d) HCC model proposed for $\mathrm{Sr} / \mathrm{Si}(111)-3 \times 2$ surface. 
Comparing our experimental STM images with theoretical results report by Lee ${ }^{[6]}$, et al, we propose that the $\mathrm{Sr} / \mathrm{Si}(111)-3 \times 2$ surface may be describe by the HCC model, which have been used to described other AEM atoms such as $\mathrm{Ca}$ and $\mathrm{Ba}$ induced $\mathrm{Si}(111)-3 \times 2$, fig.1(d) shows the model proposed for $\mathrm{Sr} / \mathrm{Si}(111)-3 \times 2$ surface. The bright area in the empty state image at $1.5 \mathrm{~V}$ reflect the $\mathrm{Sr}$ atoms position, while the double rows in the filled state image reflect the information of reconstructed $\mathrm{Si}$ atoms. Comparing with $\mathrm{AE}$ atoms induced $\operatorname{Si}(111)-3 \times 1$ surface, the $(3 \times 1)$ reconstruction of substrate silicon have some deformation, as indicated by the ellipse in the fig.1(c), the silicon atoms reacted with $\mathrm{Sr}$ atoms which denote as $d$ and $d$ ' in the model have dimeric phenomenon, in the filled state image of fig.1(c) we observe this phenomenon.

\subsection{D Diffusion of Sr Atom Chains in Sr/Si(111)-3x2surface and its Origin}

We measured two empty STM images of same area within 3min, and shown in fig.2(a) and fig.2(b), fig.2(c) was the subtraction image. After compare these two images we find that two typical changes appearing, as shown in fig.2(d): For the first type, bright rows behave obvious displacement, and the change length was varied from several (line1, 4, and line 10) to dozens of atoms (line 3 and line6); for the second type, the atom rows in one image was clear, in the other image these clear rows become blurry, and the atom row length was also varied. Besides these two type changes, there also have two type rows behave no changes: one was clear atom rows, the other was blurry atom rows. In the total image, half atom rows change, and left part kept the initial state. The above phenomenon support $\mathrm{Sr}$ atoms behave motion in the room temperature. And we will give our explanations in the following part.

First, there exist $\mathrm{Sr}$ atom vacancy defects in the $\mathrm{Sr} / \mathrm{Si}(111)-3 \times 2$ surface, $\mathrm{Sr}$ atoms nearby may move across these vacancy defects by the thermal fluctuation energy at room temperature, the selected change atom rows in fig.2(d) support this point.

Another factor comes from the lower coverage of 1/6 monolayer for $\mathrm{Sr}$ atoms. As $\mathrm{Si}$ substrate adopt the $3 \times 1$ reconstruction in $\mathrm{HCC}$ structure, there has only one $\mathrm{Sr}$ atom in a $3 \times 2$ unit cell, as two T4 sites in one unit cell have the same adsorption energy, when there have two metal atoms, the static electric force between them push the atoms away, thus these $\mathrm{Sr}$ atoms behave 1D motion phenomenon in the silicon channel.

Third, when measuring STM images, STM tip induced electric effect will accelerates the $1 \mathrm{D}$ chain motion. Besides, thermal energy at room temperature leads to the above 1D motion, which behave as the vacancy motion, and the change of atom line length in the filled state image. In the atomic resolution empty state image we can even observe $\mathrm{Sr}$ atoms moving along the silicon dimer row . 
(a)

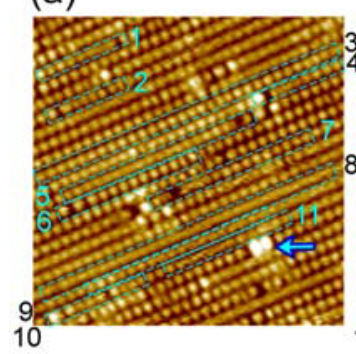

(b)

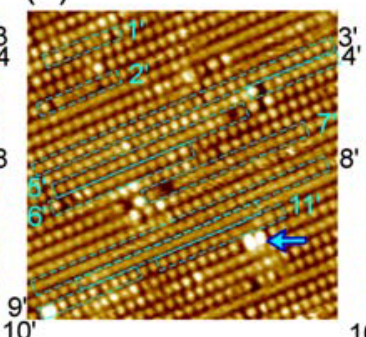

(c)

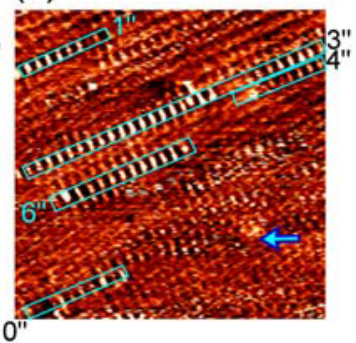

(d)

Type 1: half unit displacement

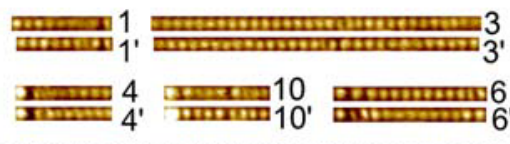

Type 2: blur and clear rows
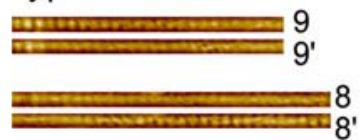

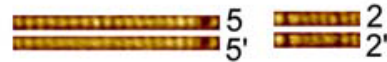

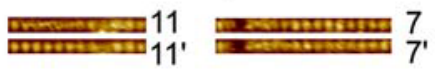

(e)

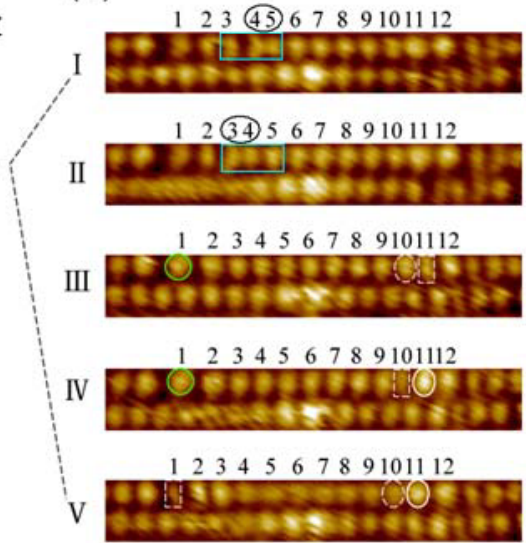

Fig.2 flip-flop phenomenon of $\mathrm{Sr} / \mathrm{Si}(111)-3 \times 2$ surface, (a) and (b) were the same area obtained sequentially within $3 \mathrm{~min}$, (c) was the image obtained by the subtraction of (a) and (b). Scanning condition of $\mathrm{V}_{\text {bias }}=3.0 \mathrm{~V}, \mathrm{I}_{\text {set }}=20 \mathrm{pA}, 22 \times 22 \mathrm{~nm}^{2}$ (d) two type changes appearing in (a) and (b). (e) Five sequenced image show the change for line 6 .

Fig.3 display a model to explain the Sr atom motion mechanism in this $1 \mathrm{D}$ diffusion.

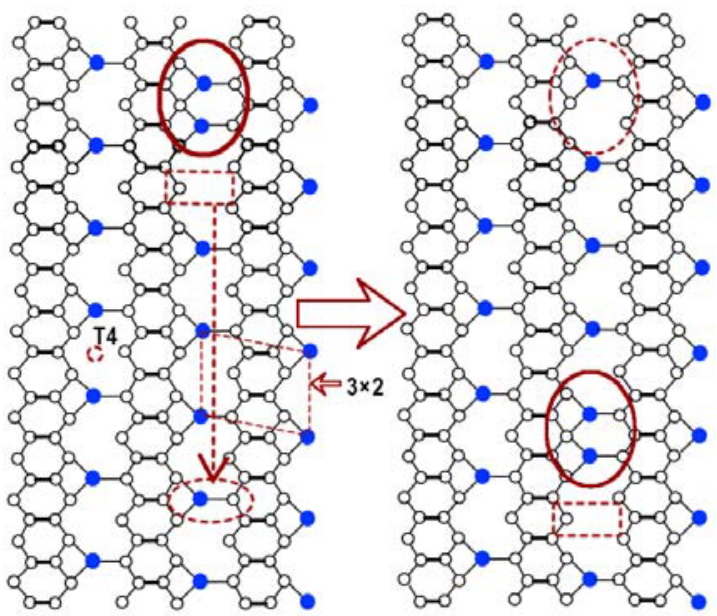

Fig.3 schematic mechanism for $\mathrm{Sr}$ atom motion along the channel formed by double bond $\mathrm{Si}$ atom 


\section{Summary}

In conclusion, $\mathrm{Sr} / \mathrm{Si}(111)-3 \times 2$ surface was investigated by $\mathrm{STM}$. As for the geometric structure, this surface can be described by HCC model with Sr coverage of 1/6 ML. Blur rows appearing in the room temperature STM images arouse from Sr atoms motion phenomenon between two adsorption sites with comparable surface energy, and this show that $\mathrm{Sr} / \mathrm{Si}(111)-3 \times 2$ surface behave the quazi-1D property.

\section{Acknowledgements}

This work was supported by National Nature Science foundation of China(NNSFC) with Grant No. of 20473077 and 61540071, Project of natural science research of high education in Jiangsu Province with Grant No. of 15KJD140002, Changzhou modern optoelectronic technology research institute funds with Grant No. of CZGY13, Natural science funds of Changzhou Institute of technology with Grant No. of YN1408.

\section{References}

1. Y.Zhou; Q. Wu; C.Zhou;H. Zhang; H.H Zhan;J.Y. Kang J. Au-induced charge redistribution on $\operatorname{Si}\left(\begin{array}{lll}1 & 1 & 1\end{array}\right)-7 \times 7$ surface, Surf. Sci. 602(2), (2008), 638-643.

2. P.G. Kang; H.Jeong; and H.W.Yeom, Hopping Domain Wall Induced by Paired Adatoms on an Atomic Wire: Si ( 111 ) - ( $5 \times 2$ ) Au, Phys.Rev. Lett. 100(14), (2008), 146103

3. F.C. Chuang; C.H.Hsu ; Z.C. Wang Honeycomb chain structure of the AuSi $(111)-(5 \times 2)$ surface reconstruction: A first-principles study, Phys. Rev. B 77(15), (2008), 153409

4. Saki Kurata and Yokoyama, Interchain coupling of degenerated quasi-one-dimensional indium chains on Si(111), Phys. Rev. B 71, (2005), 121304

5. M. Gurnett, J. B. Gustafsson, L. J. Holleboom, Core-level spectroscopy study of the Li / Si ( 111 ) $-3 \times 1, \mathrm{Na} / \mathrm{Si}$ ( 111 ) $-3 \times 1$, and K/Si ( 111 ) $-3 \times 1$ surfaces, Phys. Rev. B, 71(19), (2005), 195408

6. S. Hong, G. Lee and H. Kim Theoretical STM images of alkaline-earth metal adsorbed Si(111)3×2 surfaces, Surf. Sci. 600(18), (2006),3606

7. M.Kuzmin, R. E. Perala, P. Laukkanen High-resolution core-level photoemission study of Eu-induced $(3 \times 2)(3 \times 4)$ reconstruction on Ge(111), Phys. Rev. B 74(11), (2006) ,115320

8. W. H. Du, B. Wang, L. Xu, Z. P. Hu, X. F. Cui, B. C. Pan, J. L. Yang and G. J. Hou Identifying atomic geometry and electronic structure of $(2 \times 3)-\mathrm{Sr} / \mathrm{Si}(100)$ surface and its initial oxidation. J. Chem. Phys. 129,(2008), 164707

9. J J Yang and $\mathrm{W} H \mathrm{Du}$, One-dimensional diffusion of vacancies on an $\mathrm{Sr} / \mathrm{Si}(100)-\mathrm{c}(2 \times 4)$ surface,Chin. Phys. B 22(6) (2013),066801

10. J H He, G H Zhang, J D Guo, Q L Guo, K H Wu, Atomic structure of Sr-induced reconstructions on the Si(100) surface, J. Appl. Phys. 109 (2001),083522

11. R Zhachuk, S Teys, B Olshanetsky, and S Pereira Influence of the Si(111)-7×7 surface reconstruction on the diffusion of strontium atoms Appl. Phys. Lett. 95 (2009),061901

12. P P Agata and Mariusz Krawiec Adsorption and diffusion of atoms on the $\mathrm{Si}(335)-\mathrm{Au}$ surface, Surf. Sci. 622,(2014), 9

13. N Pawel, K Palotas, M Jalochowski and M Krawiec, Surface diffusion of $\mathrm{Pb}$ atoms on the $\mathrm{Si}(553)-\mathrm{Au}$ surface in narrow quasi-one-dimensional channels, Phys. Rev. B 89,(2014) 165426 
[1] R Zhachuk, B Olshanetsky, J Coutinho and S Pereira, Electronic effects in the formation of apparently noisy scanning tunneling microscopy images of $\mathrm{Sr}$ on $\mathrm{Si}$ ( 111 ) $-7 \times 7$, Phys. Rev. B 81, (2010), 165424 